\title{
Subfamily-Specific Specialization of RGH1/MLA Immune Receptors in Wild Barley
}

\author{
Takaki Maekawa, ${ }^{1,+}$ Barbara Kracher, ${ }^{1}$ Isabel M. L. Saur, ${ }^{1}$ Makoto Yoshikawa-Maekawa, ${ }^{1}$ \\ Ronny Kellner, ${ }^{1}$ Artem Pankin,, ${ }^{1,2}$ Maria von Korff, ${ }^{1,2}$ and Paul Schulze-Lefert ${ }^{1}$ \\ ${ }^{1}$ Max Planck Institute for Plant Breeding Research, Cologne, Germany; and ${ }^{2}$ Institute of Plant Genetics, Heinrich-Heine- \\ University and Cluster of Excellence on Plant Sciences, Düsseldorf, Germany
}

Accepted 3 October 2018.

The barley disease resistance $(R)$ gene locus mildew locus $A$ (Mla) provides isolate-specific resistance against the powdery mildew fungus Blumeria graminis hordei and has been introgressed into modern cultivars from diverse germplasms, including the wild relative Hordeum spontaneum. Known Mla disease resistance specificities to $B$. graminis hordei appear to encode allelic variants of the $R$ gene homolog 1 (RGH1) family of nucleotide-binding domain and leucine-rich repeat (NLR) proteins. Here, we sequenced and assembled the transcriptomes of $50 \mathrm{H}$. spontaneum accessions representing nine populations distributed throughout the Fertile Crescent. The assembled Mla transcripts exhibited rich sequence diversity, linked neither to geographic origin nor population structure, and could be grouped into two similar-sized subfamilies based on two major N-terminal coiled-coil (CC) signaling domains that are both capable of eliciting cell death. The presence of positively selected sites located mainly in the C-terminal leucine-rich repeats of both MLA subfamilies, together with the fact that both $\mathrm{CC}$ signaling domains mediate cell death, implies that the two subfamilies are actively maintained in the population. Unexpectedly, known MLA receptor variants that confer $B$. graminis hordei resistance belong exclusively to one subfamily. Thus, signaling domain divergence, potentially as adaptation to distinct pathogen populations, is an evolutionary signature of functional diversification of an immune receptor.

Takaki Maekawa and Barbara Kracher are joint first authors.

The RNA-Seq data generated for this study have been deposited in the National Center for Biotechnology Information Sequence Read Archive (SRA) database (BioProject accession number PRJNA432492, SRA accession number SRP132475).

${ }^{\dagger}$ Corresponding author: Takaki Maekawa;

E-mail: maekawa@mpipz.mpg.de

Funding: This work was supported by the Max-Planck-Gesellschaft and the Deutsche Forschungsgemeinschaft within the scope of the Collaborative Research Center grant SFB670 and German Cluster of Excellence on Plant Sciences (CEPLAS) EXC1028. Isabel M. L. Saur is supported by a long-term fellowship from the European Molecular Biology Organization (ALTF 368-2016).

*The $\boldsymbol{e}$-Xtra logo stands for "electronic extra" and indicates that seven supplementary figures, four supplementary text files, and four supplementary tables are published online.
Adaptation to pathogens is linked to a range of evolutionary processes that result in genetic variation and affect disease resistance traits in a host population. In the coevolutionary 'arms race' model, recurrent allele fixation in a host population is predicted to reduce genetic diversity, whereas, in the 'trench warfare' model, coexistence of functional and nonfunctional alleles is possible when a fitness penalty is associated with a functional allele in the absence of pathogens (Stahl et al. 1999; Tian et al. 2003). In plants, 'gene-for-gene' resistance (Flor 1955 ) is frequently found in interactions between hosts and hostadapted pathogens and is often associated with population-level diversification of immune receptors encoded by disease resistance $(R)$ genes (Maekawa et al. 2011a and b). The products of these $R$ genes recognize matching pathogen effectors, designated avirulence (AVR) effectors, and plants that lack matching $R$ genes are susceptible to effector-mediated pathogen virulence (Jones and Dangl 2006). A single $R$ gene can encode functionally diversified resistance alleles among individuals of the host population (Maekawa et al. 2011b). While such balancing selection at a given $R$ locus can potentially be due to coevolutionary diversification of a single $R$ and $A V R$ gene pair, in some cases, it is, rather, explained by the recognition of multiple nonhomologous effectors derived from different pathogens (Anderson et al. 2016; Karasov et al. 2014; Lu et al. 2016).

Individual $R$ genes are often members of larger gene families, are organized in complex loci of paralogous genes, and can evolve through tandem and segmental gene duplications, recombination, unequal crossing-over, and point mutations (Jacob et al. 2013). Most known $R$ genes encode intracellular nucleotide-binding domain and leucine-rich repeat (NLR) proteins. Plant NLRs belong to a subclass of the STAND (signal transduction ATPases with numerous domains) superfamily of proteins (Maekawa et al. 2011b), which possess variable $\mathrm{N}$ terminal domains, a central conserved NB-ARC (nucleotidebinding adaptor shared by APAF-1, R proteins, and CED-4). Cell death protein 4 [CED-4]) domains, and C-terminal leucine-rich repeat (LRR) regions with varying repeat numbers. Incorporation of either a TOLL/interleukin 1 receptor-like domain or a coiled-coil (CC) domain at the $\mathrm{N}$ terminus defines two major classes of NLRs, designated TNLs and CNLs, respectively. Extensive cross-plant species database searches have revealed that approximately $10 \%$ of plant NLRs additionally contain highly variable integrated domains (Ellis 2016). Effector proteins can be recognized directly by NLRs inside plant cells, in what are essentially receptor-ligand interactions (Dodds et al. 2006; Ortiz et al. 2017), or indirectly through modifications of host proteins that are associated with the NLR receptor (Axtell and Staskawicz 2003; Dodds et al. 
2006; Gutierrez et al. 2010; Mackey et al. 2002; Ntoukakis et al. 2013; Ortiz et al. 2017). During direct nonself perception, the C-terminal LRR or integrated domains are known to function as major determinants of recognition specificity for AVR effectors (Cesari et al. 2013; Dodds et al. 2006; Maqbool et al. 2015; Zhu et al. 2017). Collectively, population-level $R$ gene diversification and the diversity of NLR-mediated nonself recognition mechanisms maximize the ability of plants to cope with rapidly evolving pathogen effectors.

Powdery mildews are fungal plant pathogens that are ubiquitous in temperate regions of the world and infect nearly 10,000 species of angiosperms (Glawe 2008). Given that the powdery mildew disease caused by Blumeria graminis $\mathrm{f}$. $\mathrm{sp}$. hordei poses a significant threat to barley production (Hordeum vulgare L.), powdery mildew $R$ loci, present in germplasm collections of barley relatives including wild barley ( $H$. spontaneum L.), have been extensively investigated (Jahoor and Fischbeck 1987; Jørgensen 1994). These efforts have revealed numerous powdery mildew $R$ loci and a subset was subsequently introgressed into modern barley cultivars to genetically control the disease (Jørgensen 1994). Among these, the mildew resistance locus A (Mla) is characterized by an exceptional functional diversification; each Mla locus in a given accession confers disease resistance to a distinctive set of B. graminis hordei test isolates (races), designated Mla resistance specificity (Jørgensen 1994; Seeholzer et al. 2010). More than $30 \mathrm{~B}$. graminis hordei isolate (race)-specific resistance specificities either map to the Mla locus or to adjacent genomic regions (Jørgensen 1994). A 265-kb contiguous DNA sequence spanning the Mla locus in the barley reference cultivar Morex consists of a cluster of CNL-encoding genes belonging to three distinctive families, which are designated $R$ gene homolog (RGH)1, RGH2, and RGH3 (Wei et al. 2002). To date, 28 naturally diversified RGH1 sequences have been molecularly characterized, and the majority of these are capable of conferring isolate-specific immunity to $B$. graminis hordei (Seeholzer et al. 2010). These sequence variants appear to represent $R g h l$ alleles at the Mla locus, as evidenced by the presence of an $[\mathrm{AT}]_{\mathrm{n}}$ microsatellite in the third intron (Shen et al. 2003). However, the presence of the microsatellite has been validated for the genomic Rghl sequences of only six Mla resistance specificities to B. graminis hordei. Cultivar Morex carries a truncated and nonfunctional $R g h l$ allele, designated $R g h l b c d$, and few cultivars appear to harbor more than one functional Rghl copy (Seeholzer et al. 2010; Wei et al. 2002). Our recent work demonstrated that sequence-related MLA receptor variants recognize sequence-unrelated $B$. graminis hordei effectors via direct interaction ( $\mathrm{Lu}$ et al. 2016). In addition, the wheat Mla orthologs Sr33 (stem rust resistance 33) (Periyannan et al. 2013) and Sr50 (stem rust resistance 50) (Mago et al. 2015), introgressed from Aegilops tauschii and Secale cereale, respectively, confer resistance to the stem rust pathogen Puccinia graminis f. sp. tritici isolate Ug99, a pathogen that poses a major threat to global wheat production. These findings suggest that the last common ancestor of these cereals harbored an ancestral Mla gene and imply that Mla diversified to detect multiple nonhomologous effectors from at least two unrelated fungal pathogens, the Ascomycete B. graminis hordei and Basidiomycete Puccinia graminis f. sp. tritici.

Overexpression of the MLA, Sr33, and Sr50 N-terminal CC domains is sufficient to initiate immune signaling similar to that mediated by the corresponding full-length receptors (i.e., characterized by activation of host cell death and transcriptional reprograming for immune responses [Casey et al. 2016; Cesari et al. 2016; Jacob et al. 2018; Maekawa, et al. 2011a]). Functional analysis of MLA chimeras suggests that the LRR determines AVR recognition specificities in B. graminis hordei resistance (Shen et al. 2003). A previous study on a set of $25 \mathrm{Rghl} / \mathrm{Mla}$ cDNA sequences identified a number of residues that have been subject to positive selection and that are predicted to be located mainly at the surface-exposed concave side of the deduced LRR solenoid protein structure (Seeholzer et al. 2010). In contrast, the N-terminal CC domain is mostly invariant among the same set of receptor variants (Seeholzer et al. 2010). However, this analysis relied predominantly on known Mla resistance specificities to $B$. graminis hordei in cultivated barley and, therefore, might have underestimated $R g h l$ diversity at the Mla locus in wild barley populations.

In this study, we explored $R g h l$ sequence diversity between $H$. spontaneum accessions collected from the Fertile Crescent. In previous studies, due to sequence dissimilarity among the three $R g h$ families, $R g h l$ sequences encoding MLA receptors have been obtained either by polymerase chain reaction with gene-specific primers (Halterman and Wise 2004; Seeholzer et al. 2010) or DNA gel-blot analysis using Rghl-specific hybridization probes (Wei et al. 1999; Zhou et al. 2001). Here, we extracted transcripts belonging to the $R g h l$ family from RNA-sequencing (RNA-Seq) data collected from barley leaves. De novo transcriptome assembly of 50 wild barley accessions representing nine different wild barley populations (Pankin et al. 2018) revealed a rich sequence diversity of Mla genes that segregate into two major subfamilies. Our findings imply that the divergence of the RGH1/MLA family has been driven by subfamily-specific functional diversification.

\section{RESULTS}

Identification of Mla sequences in wild barley accessions.

To gain deeper insights into Mla diversity in wild barley populations, we used transcriptome sequencing and assembly to identify Mla sequences in a set of 50 wild barley accessions that represent nine populations distributed throughout the Fertile Crescent (Pankin et al. 2018). All accessions were purified by single-seed descent to eliminate accession heterogeneity (Pankin et al. 2018). We included six barley accessions with already-characterized Mla resistance alleles to verify that our workflow was able to correctly identify the corresponding gene transcript variants. For each accession, total mRNA was obtained from the first or second leaf at 16 to $19 \mathrm{~h}$ after inoculation with $B$. graminis f. sp. hordei conidiospores, as gene expression of Mlal, Mla6, and Mla13 was previously shown to be pathogen-inducible (Caldo et al. 2004). RNA samples were subjected to paired-end Illumina sequencing, which generated 14 to 23 million read pairs per sample. These RNA-Seq read pairs were then used for de novo assembly of transcriptomes for all accessions. Presumptive Mla transcripts were extracted from these assemblies by BLAST searches against a database of known Mla sequences (details below).

Using this workflow, we were able to efficiently recover the known Mla alleles from all six previously characterized accessions, including those from a complex case in which two Mla copies encoding polymorphic MLA variants are present in a single accession (Supplementary Fig. S1). As this initial test verified the suitability of our experimental and bioinformatics pipeline, we next applied this analysis to all wild barley accessions and were able to retrieve Mla candidates for all but five of the 50 analyzed accessions (Table 1). Expression levels of the identified Mla candidate sequences were variable, ranging from 11 to 300 FPKM (fragments per kilobase of transcript per million mapped reads) for the six previously characterized 
accessions and from 2 to 140 FPKM for the wild barley accessions (Supplementary Table S1).

Among the 45 wild barley accessions with putative Mla transcripts in 20 accessions, we reliably identified two Mla copies and, in two accessions, we even found three putative Mla copies (Table 1). This contrasts with previous findings of a single Rghl/Mla copy in 'Morex' (Wei et al. 1999, 2002), suggesting that Rgh1/Mla sequences may be duplicated or triplicated in wild barley. Five of the 69 identified transcript sequences were excluded from further downstream analysis because, in three cases, the deduced MLA proteins were C-terminally fused in-frame to a full-length RGH2 family member (i.e., RGH2-MLA), and two other deduced MLA variants lacked the $\mathrm{CC}$ domain due to truncation of the transcript at the 5' end. The three RGH2-MLA fusions (FT146-2, FT158, and FT313-2) are 99\% identical to each other at the nucleotide level, although their corresponding barley accessions belong to different populations. Moreover, for five accessions, a full-length transcript was identified but, due to a premature stop codon, the predicted proteins were truncated,

Table 1. Summary of geographic origin, population information, and identified mildew locus A (MLA) sequences for the 50 wild barley lines used in this study

\begin{tabular}{|c|c|c|c|c|c|c|c|c|c|c|c|}
\hline Sample & Line_ID & Origin $^{a}$ & Latitude & Longitude & Population & $\begin{array}{c}\text { No. of } \\
\text { Rgh1/Mla }\end{array}$ & $\begin{array}{c}\text { Identity } \\
\text { between } \\
\text { sequences }(\%)\end{array}$ & $\begin{array}{c}\text { Subfamily } \\
1\end{array}$ & $\begin{array}{c}\text { Subfamily } \\
2\end{array}$ & Other & $\begin{array}{c}\text { In } \\
\text { phylogeny }\end{array}$ \\
\hline FT010 & B1K-04-08 & ISR & 31.834371 & 35.305292 & $\mathrm{JJ}$ & - & - & - & - & - & - \\
\hline FT012 & B1K-04-13 & ISR & 31.834361 & 35.304309 & $\mathrm{JJ}$ & 1 & - & 1 & - & - & Yes \\
\hline FT013 & B1K-05-11 & ISR & 31.926131 & 35.469146 & $\mathrm{JJ}$ & 2 & 85.9 & 1 & - & 1 & Yes \\
\hline FT017 & B1K-06-07 & ISR & 32.068642 & 35.398412 & JJ & 2 & $98.1^{b}$ & - & 2 & - & Yes \\
\hline FT021 & B1K-07-02 & ISR & 32.342164 & 35.515748 & $\mathrm{JJ}$ & - & - & - & - & - & - \\
\hline FT041 & B1K-11-05 & ISR & 30.889786 & 34.631073 & NM & 1 & - & 1 & - & - & Yes \\
\hline FT045 & B1K-12-06 & ISR & 31.672915 & 35.436719 & $\mathrm{JJ}$ & 1 & - & 1 & - & - & Yes \\
\hline FT048 & B1K-13-01 & ISR & 32.852183 & 35.680149 & $\mathrm{GH}$ & - & - & - & - & - & - \\
\hline FT057 & B1K-15-07 & ISR & 32.6906 & 35.662224 & $\mathrm{GH}$ & 2 & $95.4^{\mathrm{b}}$ & 2 & - & - & Yes \\
\hline FT086 & B1K-21-08 & ISR & 32.647805 & 34.967268 & $\mathrm{CG}$ & 2 & 90.1 & 1 & 1 & - & Yes \\
\hline FT087 & B1K-21-11 & ISR & 32.647599 & 34.967754 & $\mathrm{CG}$ & 3 & $89.5 / 89.8 / 95.7^{b}$ & 2 & 1 & - & Yes \\
\hline FT088 & B1K-21-20 & ISR & 32.647674 & 34.966946 & $\mathrm{CG}$ & 2 & 89.6 & 1 & 1 & - & Yes \\
\hline FT113 & B1K-27-02 & ISR & 32.875386 & 35.544077 & $\mathrm{HG}$ & 2 & 85.9 & - & 1 & 1 & $\begin{array}{l}\text { Only } \\
\text { FT113-1 }\end{array}$ \\
\hline FT115 & B1K-27-11 & ISR & 32.874543 & 35.54338 & $\mathrm{HG}$ & 1 & - & 1 & - & - & Yes \\
\hline FT120 & B1K-28-10 & ISR & 32.823909 & 35.497509 & $\mathrm{HG}$ & 1 & - & - & 1 & - & Yes \\
\hline FT125 & B1K-29-20 & ISR & 32.821027 & 35.507253 & $\mathrm{HG}$ & 1 & - & - & - & 1 & Yes \\
\hline FT132 & B1K-31-01 & ISR & 32.773355 & 35.44657 & NM & 2 & $95.0^{\mathrm{b}}$ & 2 & - & - & Yes \\
\hline FT138 & B1K-32-08 & ISR & 32.578564 & 35.471275 & NM & 2 & 89.7 & 1 & 1 & - & Yes \\
\hline FT145 & B1K-33-19 & ISR & 30.571722 & 34.676408 & $\mathrm{HG}$ & - & - & - & - & - & - \\
\hline FT146 & B1K-34-04 & ISR & 30.845141 & 34.744956 & $\mathrm{NM}$ & 2 & 84.7 & 1 & - & 1 & $\begin{array}{l}\text { Only } \\
\text { FT146-1 }\end{array}$ \\
\hline FT152 & B1K-35-04 & ISR & 31.596864 & 34.898841 & SCJ & 1 & - & 1 & - & - & Yes \\
\hline FT153 & B1K-35-08 & ISR & 31.596249 & 34.898955 & SCJ & 1 & - & 1 & - & - & Yes \\
\hline FT158 & B1K-36-05 & ISR & 32.925892 & 35.531067 & $\mathrm{HG}$ & 1 & - & - & - & 1 & No \\
\hline FT169 & B1K-39-02 & ISR & 33.070336 & 35.769545 & $\mathrm{GH}$ & 1 & - & 1 & - & - & Yes \\
\hline FT170 & B1K-39-20 & ISR & 33.070844 & 35.769422 & $\mathrm{GH}$ & 2 & 90.1 & 1 & 1 & - & Yes \\
\hline FT174 & B1K-41-03 & ISR & 33.251503 & 35.647858 & SCJ & 2 & $95.5^{\mathrm{b}}$ & 2 & - & - & Yes \\
\hline FT218 & B1K-49-18 & ISR & 31.771952 & 35.112916 & SCJ & 1 & - & - & - & 1 & Yes \\
\hline FT231 & HID-4 & IRQ & 35.58333333 & 43 & UM & 1 & - & - & 1 & - & No \\
\hline FT233 & HID-8-1 & IRQ & 36.41666667 & 41.65 & UM & 1 & - & - & 1 & - & No \\
\hline FT248 & HID-53 & IRN & 31.67589444 & 48.57915556 & LM & 1 & - & - & - & 1 & Yes \\
\hline FT256 & HID-65 & TUR & 36.75194444 & 37.47527778 & UM & 1 & - & - & 1 & - & No \\
\hline FT271 & HID-104 & SYR & 36.77194444 & 40.86194444 & UM & 1 & - & - & 1 & - & No \\
\hline FT281 & HID-137 & TUR & 36.85083333 & 40.0475 & UM & 2 & 89.7 & 1 & 1 & - & Yes \\
\hline FT282 & HID-138 & IRN & 32 & 48.55 & LM & - & - & - & - & - & - \\
\hline FT283 & HID-140 & IRQ & 36.4 & 44.2 & UM & 2 & 89.3 & 1 & 1 & - & Yes \\
\hline FT287 & HID-145 & ISR & 31 & 34.91666667 & NM & 2 & 89.7 & 1 & 1 & - & Yes \\
\hline FT289 & HID-149 & ISR & 32.96666667 & 35.6 & $\mathrm{GH}$ & 1 & - & - & 1 & - & Yes \\
\hline FT293 & HID- 160 & ISR & 31.6 & 34.9 & $\mathrm{GH}$ & 2 & $95.5^{\mathrm{b}}$ & 2 & - & - & Yes \\
\hline FT313 & HID-213 & ISR & 31.4 & 34.6 & SCJ & 2 & 85.6 & 1 & - & 1 & $\begin{array}{l}\text { Only } \\
\text { FT313-1 }\end{array}$ \\
\hline FT320 & HID-230 & ISR & 32.25 & 34.85 & SCJ & 2 & 89.9 & 1 & 1 & - & Yes \\
\hline FT349 & HID-302 & IRN & 32.55 & 48.81666667 & LM & 3 & $89.7 / 89.6 / 95.5^{\mathrm{b}}$ & 2 & 1 & - & Yes \\
\hline FT352 & HID-307 & IRN & 31.53333333 & 48.81666667 & LM & 2 & 90.6 & 1 & 1 & - & Yes \\
\hline FT353 & HID-308 & IRN & 31.53333333 & 48.81666667 & LM & 1 & - & 1 & - & - & Yes \\
\hline FT355 & HID-310 & IRN & 31.53333333 & 48.81666667 & LM & 2 & 86.0 & - & 1 & 1 & $\begin{array}{l}\text { Only } \\
\text { FT355-1 }\end{array}$ \\
\hline FT393 ${ }^{c}$ & n.d. & n.d. & n.d. & n.d. & n.d. & 1 & - & 1 & - & - & Yes \\
\hline FT394 ${ }^{c}$ & HID-386 & ISR & 31.7747333 & 35.0485083 & $\begin{array}{l}\text { SCJ } \\
\quad(\text { mixed })\end{array}$ & 1 & - & - & 1 & - & Yes \\
\hline FT454 & HP-10-4 & TUR & 36.2558333 & 36.4672222 & NL & 1 & - & 1 & - & - & Yes \\
\hline FT458 & HP-15-1 & TUR & 37.49333333 & 38.87666667 & NL & 1 & - & 1 & - & - & Yes \\
\hline FT462 & HP-20-1 & TUR & 37.77833333 & 39.78 & NL & 2 & 89.0 & 1 & 1 & - & $\begin{array}{l}\text { Only } \\
\text { FT462-2 }\end{array}$ \\
\hline FT471 & HP-34-3 & TUR & 37.70222222 & 38.01222222 & NL & 1 & - & 1 & - & - & Yes \\
\hline
\end{tabular}

${ }^{a}$ ISR = Israel, IRQ = Iraq, IRN = Iran, TUR = Turkey, and SYR = Syria; n.d. = not determined.

b The level of sequence similarity between co-occurring Rgh1/Mla sequences belonging to the same subfamily.

${ }^{c}$ FT393 and FT394 are also known as ISR42-8 and ISR101-23, respectively. Taxonomy of FT393 is not determined (n.d.). 
lacking part of the NB-ARC domain and the complete LRR. These five sequences were also not included in the phylogenetic analyses unless otherwise stated (Table 1). Furthermore, we were unable to detect any apparent integrated domains other than the CC, NB-ARC, and LRR among the deduced protein sequences.

\section{Phylogenetic analysis of MLA sequences.}

We performed multisequence alignment (MSA) and subsequent phylogenetic analysis on the 59 full-length candidate MLA protein sequences retrieved from wild barley together with the 28 previously characterized full-length MLA proteins (Seeholzer et al. 2010) (excluding truncated MLA38-1) as well as the MLA homologs TmMLA1 from wheat (Jordan et al. 2011), Sr33 from wheat (Periyannan et al. 2013), Sr50 introgressed to wheat from Secale cereale (Mago et al. 2015), and the closest homolog to MLA from the more distantly related Brachypodium distachyon (XP_014754701.1). The MSA showed that one wild barley accession (FT170) contains the two previously characterized Mla alleles Mla18-1 and Mla18-2, whereas two accessions (FT394 and FT355) contain the known Mla25-1 (Fig. 1A; Supplementary Fig. S2). Similarly, in one accession (FT113), one of the two deduced MLA variants differs from already characterized MLA25-1 by only two amino acids. In another accession (FT313) the aligned sequence of one of the two deduced MLA variants is identical to MLA34 but the wild barley sequence shows an extension in the LRR (Fig. 1A). These results further confirmed that our method could be used to efficiently recover natural Mla variants in wild barley. Our findings indicate that the sequences of at least a few previously published Mla alleles have been conserved in extant wild barley populations after their introgression into modern barley cultivars.

Overall, the phylogenetic analysis revealed that the newly identified RGH1/MLA proteins from the wild barley accessions do not constitute a distinct clade but are rather distributed across the phylogeny together with known MLA variants conferring B. graminis hordei resistance (Jordan et al. 2011; Seeholzer et al. 2010) (Fig. 1A). We conclude that these Mla transcripts, retrieved from the wild barley accessions, are derived from naturally occurring Mla variants. However, because of markedly lower sequence similarity to the others, two sequences, FT125 and FT013-2, might be derived from an $R G H$ family other than RGHI (Fig. 1; Table 1). The phylogeny of MLA sequences does not show an obvious relation to the population of origin of the corresponding accessions, while a set of 13 known Mla resistance specificities to B. graminis hordei (e.g., Mla6, Mla7, and Mla9) cluster in the phylogenetic tree (Fig. 1A). To crosscheck the results of the neighbor-joining (NJ) phylogenetic analysis, we additionally generated a maximum likelihood (ML) tree from the same data and compared the two phylogenies (Supplementary Fig. S3). As this comparison showed a good agreement between the two methods, further analyses were performed using the less computationally intensive NJ approach.

When including the five C-terminally truncated sequences in the phylogenetic analysis, we found that four of the five sequences with premature stop codons are identical to each other (Supplementary Fig. S4A). While the four corresponding barley accessions belong to the same UM population, this population also includes accessions harboring different MLA sequences (Fig. 1A). Nevertheless, this is the only example in which a wild barley population (i.e., the UM population) is seemingly dominated by an invariant RGH1/MLA coding sequence.

A BLAST search of the recently updated barley genome annotations (Mascher et al. 2017) detected, in addition to the previously described Rghlbcd copy (Wei et al. 2002), another potential Rghl/Mla copy (HORVU1Hr1G012190) encoding a protein with a slightly truncated LRR in the genome of 'Morex'. Although no transcript corresponding to this gene is detected in tissues (using the IPK Barley BLAST server), an $[\mathrm{AT}]_{\mathrm{n}}$ microsatellite is found in the third intron, which is commonly found in the six previously isolated Mla resistance alleles functioning in B. graminis hordei resistance (Shen et al. 2003). Based on the genome of 'Morex' (Mascher et al. 2017), the corresponding gene would be located approximately $22 \mathrm{Mb}$ downstream of the previously reported Mla locus (Wei et al. 2002), suggesting that Rghl/Mla family members can be encoded at distant locations in the barley genome.

\section{Exclusive deployment of one MLA subfamily in modern barley cultivars for $B$. graminis hordei resistance.}

For a more detailed examination of MLA sequence diversity, we focused on the three functional domains of MLA and performed phylogenetic analyses for each domain separately. In agreement with a previous report using mainly barley cultivars (Jordan et al. 2011), our extended dataset detected two distinct subfamilies in the wild barley accessions that can be defined by two distinct $\mathrm{CC}$ domain sequences (Fig. 1A and B). Although the bootstrap support for discrimination of the two distinct CC domain subfamilies in the NJ tree (Fig. 1A) is not very high (74\%), neighbor-net analysis provided supporting evidence with very high bootstrap support ( $>95 \%$ ) (Fig. 1C and D). This result suggests that the underlying evolutionary history of Rghl/Mla is not tree-like. Furthermore, we found a striking pattern of $\mathrm{CC}$ sequence diversification, characterized by differential occupancy of charged or noncharged amino acids in subfamilies 1 and 2 (Supplementary Fig. S5). These charge alterations ultimately distinguish the two MLA/RGH1 subfamilies. Thirty-nine $(61 \%)$ of the 64 MLA sequences from wild barley (including the five $\mathrm{C}$-terminally truncated proteins) belong to CC domain subfamily 1 and $39 \%$ to subfamily 2 and, in several accessions, we observed the co-occurrence of both subfamilies (Table 1). Within the 64 novel sequences and 29 known MLA sequences, we can distinguish 27 sequence haplotypes based on protein sequences for the $\mathrm{CC}$ domain, of which 16 belong to subfamily 1 and nine to subfamily 2 (Fig. 1E; Supplementary Table S2) Notably, all known MLA variants conferring resistance to $B$. graminis hordei belong exclusively to subfamily 1 . Furthermore, the most common CC haplotype (haplotype $\mathrm{CC} 01$ ) for the MLA variants providing $B$. graminis hordei resistance (13 of 25 variants) is found only in three wild barley accessions (FT170-2, FT458, and FT471). These data suggest that the CC haplotype in subfamily 1 might effectively confer resistance to $B$. graminis horde $i$ in agricultural settings and that this haplotype has predominantly been deployed in modern barley cultivars.

As for the full-length and $\mathrm{CC}$ domain sequences, $\mathrm{NJ}$ phylogenetic and neighbor-net analyses based on the NB-ARC domain and LRR showed that many clades contain the reported MLA sequences and sequences from wild accessions (Fig. 2A to D). We distinguished a total of 44 sequence haplotypes for the NB-ARC domain (Fig. 1E). Among them, NB-ARC domains of 12 MLA variants conferring B. graminis hordei resistance constitute the most common haplotype together with those of FT458 and FT313-1 (Fig. 2A and C). While the clear separation into the two MLA subfamilies is partly retained for the NB-ARC domain (Fig. 2C), the LRR does not contribute to the subfamily grouping (Fig. 2D). Moreover, pair-wise comparisons of the phylogenies for the three domains show that, while certain smaller phylogenetic groups might be conserved across domains, major discrepancies can be detected, especially between the LRR phylogeny and the NB-ARC and CC 


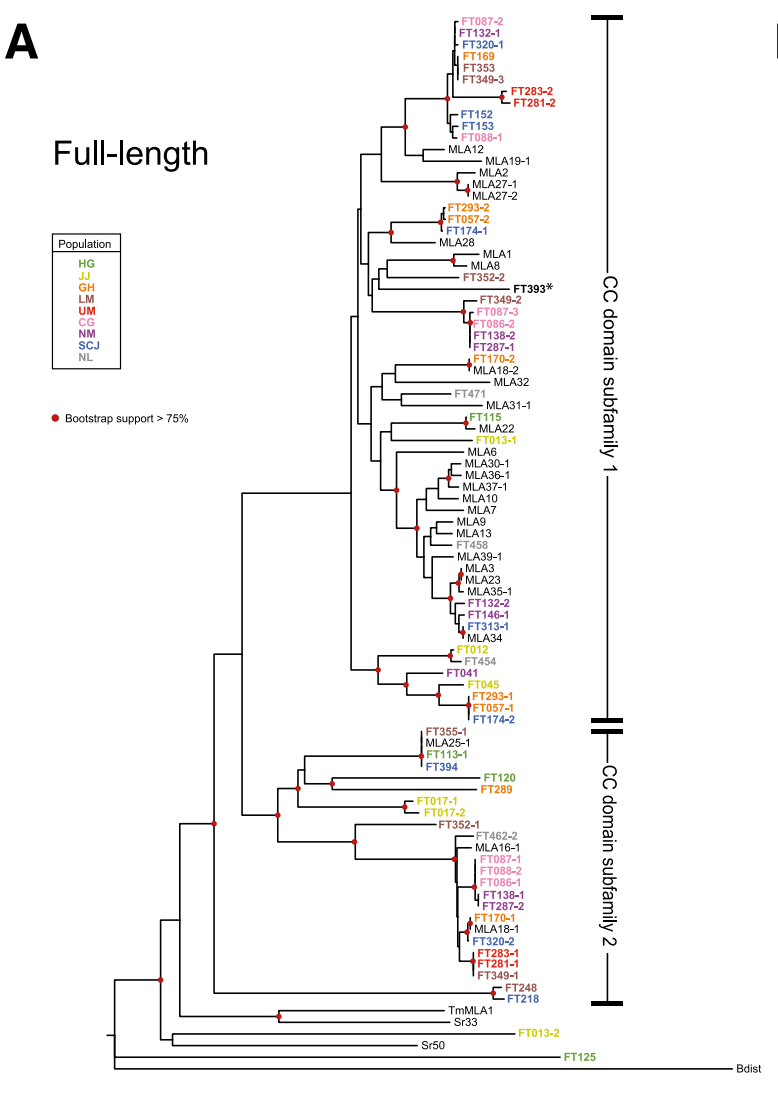

B
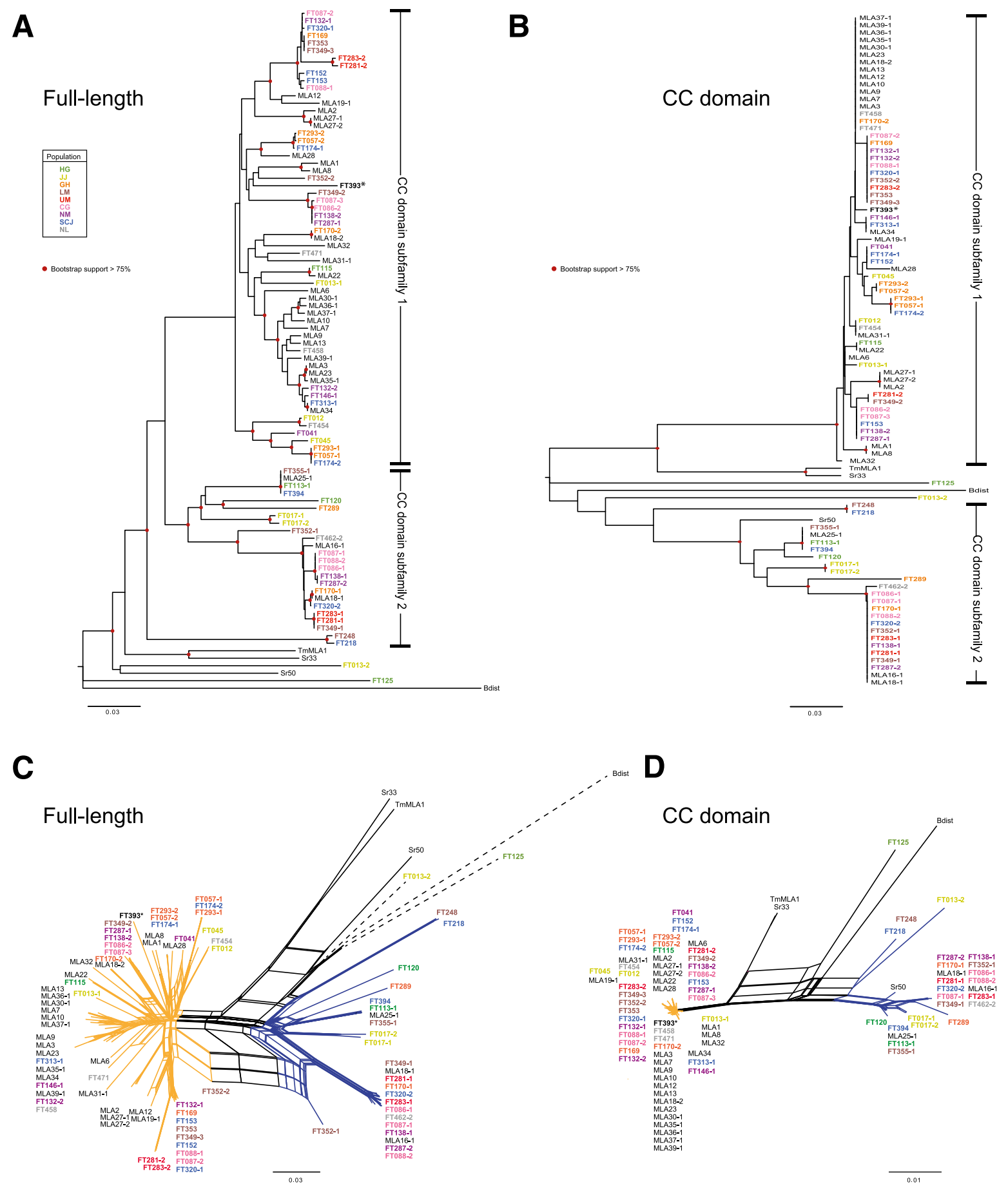

E

$$
\begin{array}{cccc}
\multicolumn{4}{c}{\text { Number of haplotypes of } 87 \text { MLA sequences }} \\
\hline \text { CC } & \text { NB-ARC } & \text { LRR } \\
\hline 27 & 44 & 67 & \text { at } 100 \% \text { identity } \\
7 & 10 & 48 & \text { at } 95 \% \text { identity } \\
\hline
\end{array}
$$

Fig. 1. Phylogenetic analysis of 91 mildew locus A (MLA) and MLA-like protein sequences. A, Unrooted neighbor-joining (NJ) tree of full-length proteins; B, $\mathrm{NJ}$ tree of the CC (coiled-coil) domain (amino acids [aa] 1 to 151); $\mathbf{C}$, neighbor-net (NN) analysis of full-length proteins; and $\mathbf{D}$, NN analysis of the CC domain (aa 1 to 151). These analyses were conducted using 28 previously published MLA protein sequences from barley (indicated in black) (Seeholzer et al. 2010), four MLA homologs from other species (indicated in black), and 59 candidate MLA sequences, identified in this study, from 50 wild barley accessions (colored by population of origin). Only MLA sequences harboring all three domains with more than 895 aa were used in this analysis. In A and B, red circles mark branches with bootstrap support $>0.75$ (500 bootstrap replicates). In C and D, the protein sequences indicated by orange and blue edges are separately grouped with bootstrap support $>0.95$ (1,000 bootstrap replicates). The branch length of dashed lines is reduced to half of the actual length. E, Number of haplotypes based on protein sequences of the CC and NB-ARC (nucleotide-binding adaptor shared by APAF-1, R proteins, and CED-4)) domains and leucine-rich repeat (LRR) regions of 87 barley MLA sequences found in this and previous studies. No Blumeria graminis hordei resistance activity was detected for MLA16-1, MLA18-1, and MLA25-1 (Seeholzer et al. 2010). The population information of FT393 is unavailable. 
A

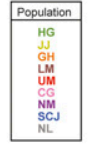

4 CC domain subtamily 2

- Bootstrap support $>75 \%$

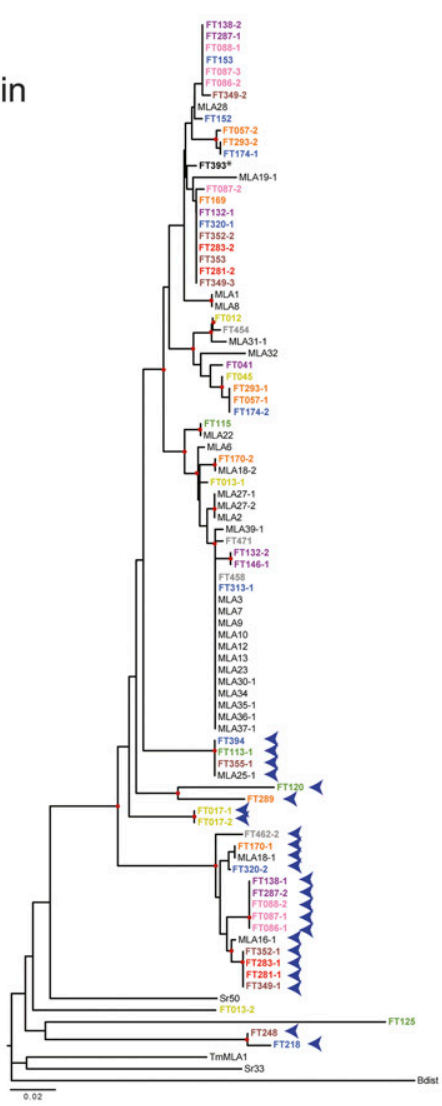

C

NB-ARC domain

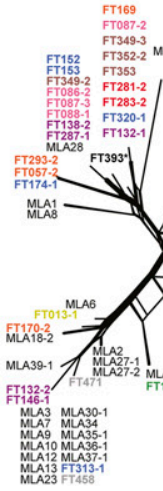

B

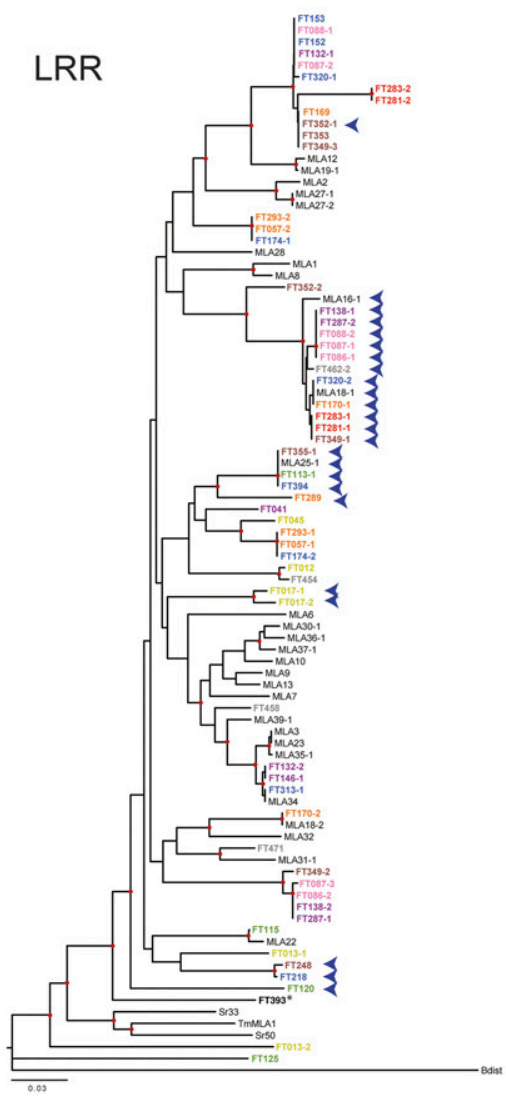

LRR 
phylogenies. This suggests that the three domains, especially the LRR, have evolved largely independently from each other.

\section{Most sites under positive selection locate to the LRR.}

To estimate MLA sequence diversity, we excluded the two most-divergent candidate sequences (FT125 and FT013-2), as we could not exclude that these represent RGH proteins other than RGH1. Based on the remaining 57 full-length candidate MLA sequences and the 28 previously published full-length MLA variants, we observed an overall nucleotide diversity $(\pi)$ of 0.068. Sequence diversity within the wild accessions $(\pi=$ 0.072 ) is slightly higher than in the previously published MLA variants $(\pi=0.055)$ and sequence diversity is also higher in subfamily $2(\pi=0.060)$ compared with subfamily $1(\pi=0.045)$ (Supplementary Table S3).

The pair-wise comparisons of the phylogenies for the three MLA domains demonstrate highly domain-specific sequence selection within a single gene. Thus, we performed dedicated statistical analyses on the coding sequences of the 85 fulllength MLA sequences to identify sites under episodic (MEME [a mixed effects model of evolution]) or pervasive (FUBAR [fast unconstrained Bayesian approximation]) positive selection. The former analysis was included to also allow detection of positive selection sites acting on a subset of branches in a phylogeny. In accordance with previous observations (Seeholzer et al. 2010), most sites under positive selection,
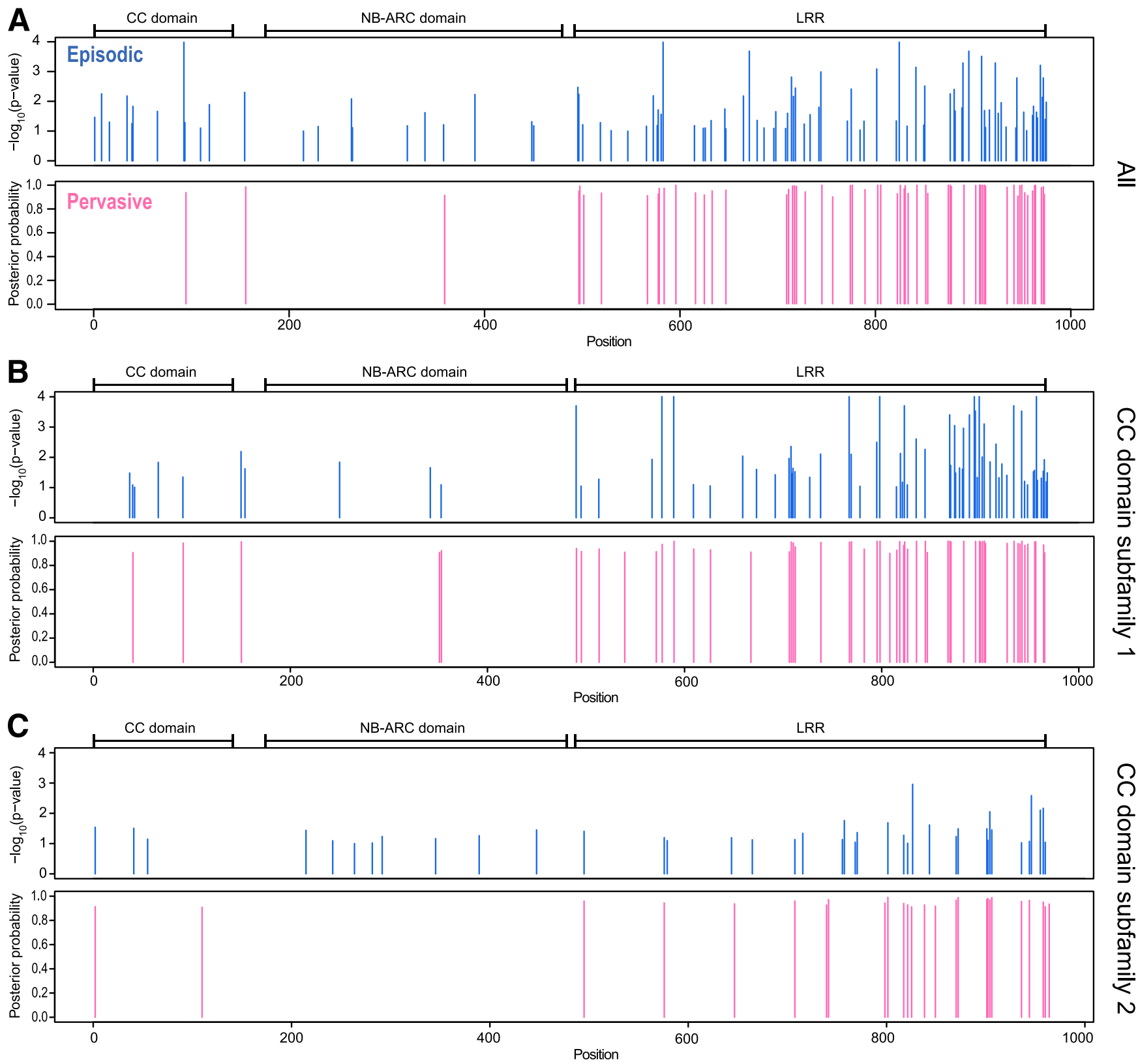

Fig. 3. Identification of positively selected sites in previously known and newly identified candidate mildew locus A (MLA) cDNAs. A, Sites under episodic (upper panel, blue bars) or pervasive (lower panel, pink bars) positive selection in a set of 85 known and newly identified MLA cDNAs. B, Sites under episodic (upper panel, blue bars) or pervasive (lower panel, pink bars) positive selection in 61 known and newly identified MLAs carrying a coiled coil (CC) domain belonging to subfamily 1. C, Sites under episodic (upper panel, blue bars) or pervasive (lower panel, pink bars) positive selection in 24 known and newly identified MLAs carrying a CC domain belonging to subfamily 2. Only full-length MLA sequences (at least 895 amino acids [aa]) from barley were included in these analyses. To test for episodic selection, we used MEME (a mixed effects model of evolution) and judged all sites with a $P$ value below 0.1 to be under positive selection. To test for pervasive selection, we additionally used FUBAR (fast unconstrained Bayesian approximation) and judged all sites with a posterior probability above 0.95 to be under positive selection. CC domain: aa 1 to 151; NB-ARC domain: aa 180 to 481; LRR region: aa 490-end. 
whether pervasive or episodic, are located in the LRR (Fig. 3A). Using this complete dataset, we also observed a number of sites under episodic positive selection $(P<0.1)$ in the $\mathrm{CC}$ domain, which mostly involve residues that differ between the two subfamilies (Fig. 3A). Accordingly, when we performed separate analyses for the two subfamilies, we observed that, within each subfamily, only a very few sites under positive selection are in the $\mathrm{CC}$ or NB-ARC domains (Fig. 3B and C). In these separate analyses for both subfamilies, sites under pervasive positive selection (posterior probability $>0.95$ ) are detected almost exclusively in the LRR, and many of these sites in the LRR seem to be under positive selection within both subfamilies as well as in the complete set (Fig. 3). The presence of additional sites under episodic positive selection in subfamilies 1 and 2 suggests distinct selection pressures acting on a few branches of both subfamilies (Fig. 3B and C). The location and clustering of sites under positive selection for subfamily 1 is largely conserved between the sequences from wild barley and known MLA resistance specificities to $B$. graminis hordei (Supplementary Fig. S7). Among 18 sites for the wild accessions and 16 sites for known MLA resistance specificities to $B$. graminis hordei that are located in the $\beta$-strand motifs in the LRR, 13 sites are shared.

The subfamily $2 \mathrm{CC}$ domain is functional in cell death.

The ability of RGH1 members from subfamily 2 to confer resistance to $B$. graminis hordei remains unclear, as no $B$. graminis hordei resistance activities were detected for MLA16-1, MLA18-1, and MLA25-1 (Jordan et al. 2011; Seeholzer et al. 2010). This raises the question of whether subfamily 2 MLA receptors can generally act as disease resistance proteins. Our phylogenetic analyses of MLA CC domains allowed us to detect a close relationship between $\mathrm{Sr} 50$ (conferring resistance to Puccinia graminis f. sp. tritici in wheat) and subfamily 2 MLAs (Fig. 1B and D). Overexpression of MLA10 representing the haplotype CC01, Sr33, and Sr50 Nterminal $\mathrm{CC}$ domains initiates downstream signaling events that are comparable to the signaling mediated by the corresponding full-length receptors, including the execution of cell death (Casey et al. 2016; Cesari et al. 2016; Jacob et al. 2018; Maekawa, et al. 2011a and b). We thus examined whether subfamily 2 MLA CC domain variants are capable of triggering cell death in planta. Overexpression of the CC domain of FT394, one of the closest barley variants of wheat Sr50, was able to induce cell death in Nicotiana benthamiana leaves (Fig. 4), suggesting that Mla alleles in subfamily 2 encode receptors with cell death activity. Although the FT394 CC variant is 93 and $67 \%$ identical to the CC domains of Sr50 and MLA10, respectively, the time of onset and confluence of the necrotic lesions induced by FT394 CC and MLA10 CC were comparable, whereas lesions elicited by the expression of $\mathrm{Sr} 33$ and Sr50 CC domains remained invariably patchy.

\section{The Sr33 CC domain harbors unique amino acid substitutions.}

In contrast to $\mathrm{Sr} 50, \mathrm{Sr} 33$ is assigned to a clade of the $\mathrm{CC}$ domain together with TmMLA1 (Fig. 1B and D). Notably, interspecies comparison of MLA or MLA-like sequences identified unique amino acid polymorphisms in the $\mathrm{Sr} 33$ and TmMLA1 CC-domains. At position 21 of the CC domains of MLA functioning in B. graminis hordei resistance, MLA from wild barley and homologs of some Triticeae family members are generally occupied by aspartate or glutamate, but glycine occupies the corresponding positions in Sr33 and TmMLA1. The same substitution is found in an accession of rye (Secale cereale). The structure of the Sr33 CC domain (6 to 120 amino acids) adopts a monomeric four-helix bundle conformation, while the structure of the MLA10 CC domain (5 to 120 amino acids) is arranged in an antiparallel homodimer that adopts a helix-loop-helix fold (Casey et al. 2016; Maekawa et al. 2011a). Intriguingly, in the corresponding structures, position 21 of MLA10 locates to the middle of the alpha helix, while in Sr33 this position with an adjacent valine corresponds to a loop region of the CC domain. These amino acid differences might account for the differences in tertiary protein structure between the CC domains of MLA10 and Sr33.

\section{DISCUSSION}

Divergence of Mla subfamilies in wild barley populations.

We revealed a rich sequence diversity of the Rghl/Mla gene family in wild barley accessions representing nine populations distributed throughout the Fertile Crescent in the Middle East. In addition, in wild barley Rghl/Mla sequences can be duplicated or triplicated (Table 1), necessitating a revision of the current view that Mla recognition specificities to B. graminis hordei represent alleles of a single gene (Shen et al. 2003). We found no clear evidence for geographic isolation and population structure of particular Mla sequence clades. Similarly, a genome-wide survey of NLR genes in Arabidopsis thaliana revealed no evidence for regional selection of particular disease resistance genes (Bakker et al. 2006). The observed similar ratio of Mla subfamily 1 and 2 members among individual wild barley accessions (37 and 27, respectively [Table 1]) was
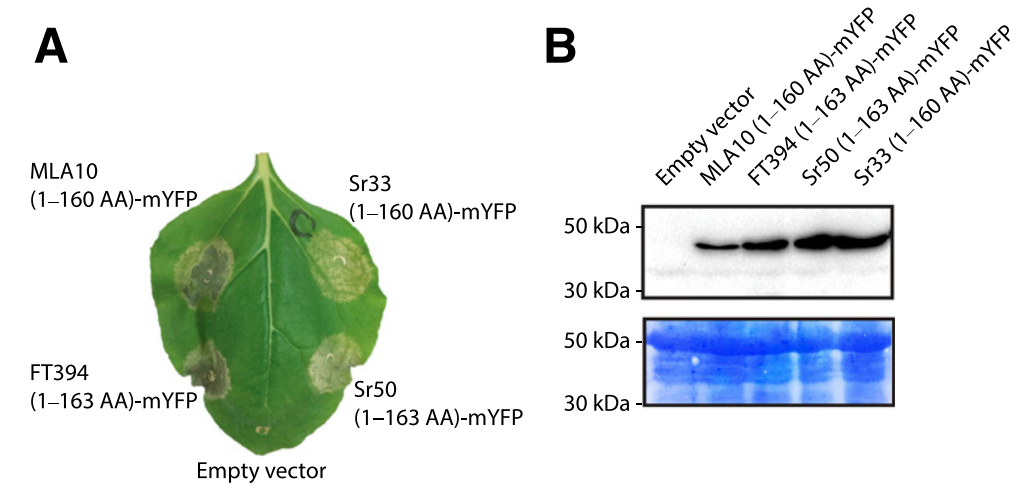

Fig. 4. The coiled coil (CC) domain of subfamily 2 mildew locus A (MLA) FT394 exhibits cell-death activity. A, Nicotiana benthamiana plants were transiently transformed to express the CC domains of MLA10_1-160AA, FT394_1-163AA, Sr50_1-163AA, and Sr33_1-160AA, each fused C-terminally to monomeric yellow fluorescent protein (mYFP) or empty vector control; the picture was taken 3 days postinfiltration. B, Immunoblot analysis corresponding to A. Transformed leaf tissue was harvested $24 \mathrm{~h}$ postinfiltration; proteins were analyzed after gel electrophoresis and Western blotting with an anti-green fluorescent protein antibody. 
unexpected and suggests that a balancing selection mechanism maintains the two subfamilies in the host populations. Future analysis of the genomic sequences adjacent to each subfamily member will reveal the degree of the presumptive balancing selection. Pathogen selection pressure is likely driving the observed sequence diversification, at least within subfamily 1 , because all known Mla resistance specificities to $B$. graminis hordei belong to this subfamily (Jordan et al. 2011; Seeholzer et al. 2010). In comparison with the $\mathrm{CC}$ domain, sequences of the LRR do not group into two subfamilies (Fig. 2D). This contrasting pattern indicates that selection regimes differ between the CC and LRR domains that might involve recombination events that swap LRRs between two Rghl/Mla alleles in the two different subfamilies. A quantitative trait loci analysis on disease resistance in the field, conducted in a cross derived from the barley cultivar Carola and the wild barley accession FT394, which carries only one subfamily 2 member with a functional CC domain (Table 1; Fig. 4), did not reveal any powdery mildew resistance mediated by the Mla locus (Wang 2005). In addition, the Mla locus did not associate with any of 13 tested agronomic traits in a population derived from a cross between barley cultivar Apex and FT394, suggesting that this Mla allele does not affect agronomic performance in the field (Pillen et al. 2003). Thus, these data reject the hypothesis that introgression of subfamily 2 in domesticated barley has been subject to strong counterselection. Conservation of NLR motifs needed for immune receptor function (Rairdan et al. 2008; Tameling et al. 2006), together with positively selected sites in the LRR among subfamily 2 members (Fig. 3C), implies a similar functional diversification of subfamily 2 NLRs in response to one or more barley pathogens. Whether subfamily 2 NLRs confer disease resistance to $A V R$ genes present in yet-uncharacterized $B$. graminis hordei populations or other pathogens remains to be tested. The simultaneous presence of both subfamily 1 and 2 members in the genomes of several accessions (14 of 50 accessions) (Table 1) is also suggestive of nonredundant immune functions of the two subfamilies.

The CC domain sequences of barley subfamily 2 members are more closely related to that of $\mathrm{Sr} 50$ than those from subfamily 1, which is encoded by an Mla ortholog originating from rye (Secale cereale) and which was introgressed into wheat for resistance against the stem rust fungus Puccinia graminis f. sp. tritici (Mago et al. 2015). Rye and wheat diverged from each other approximately three to four million years ago and barley diverged from wheat eight to nine million years ago (Krattinger and Keller 2016). Closer inspection of the phylogenetic tree of MLA CC domains indicates that the subfamily $2 \mathrm{CC}$ domain is evolutionarily maintained, at least in rye and barley (Fig. 1B and D). In contrast, the presence of subfamily 1 members appears to be restricted to barley (Fig. $1 \mathrm{~B}$ and D). The CC domains of both barley MLA subfamilies are capable of cell-death initiation, further supporting our hypothesis that members belonging to subfamily 2 also encode NLRs mediating innate immunity. The MLA subfamilies are mainly defined by two major haplotypes of the $\mathrm{N}$-terminal $\mathrm{CC}$ signaling domain rather than the C-terminal LRR that has been shown to determine AVR recognition specificities in $B$. graminis hordei resistance (Figs. 1 and 2) (Shen et al. 2003). What evolutionary mechanism has maintained the two barley MLA subfamilies in the host population? To date, only MLA subfamily 1 members have been shown to confer immunity to the barley powdery mildew fungus (Seeholzer et al. 2010; Jordan et al. 2011). One possible explanation is that B. graminis hordei has overcome immune signaling mediated by the $\mathrm{CC}$ of subfamily 2 members. For example, the effectors of the Puccinia graminis f. sp. tritici stem rust pathogen can suppress cell death mediated by the
Sr50 CC domain when overexpressed (Chen et al. 2017). However, we did not detect a markedly higher incidence of positive selection within the CC domain of subfamily 2 (Fig. 3C), which could be mediated by an ongoing coevolutionary process between host and pathogen. Considering the highly similar CC domain sequences within each subfamily, evolutionary pressure acting on the $\mathrm{CC}$ domain might be currently very low in the wild barley population or not detectable as a higher rate of nonsynonymous substitutions within the CC domain. Thus, the underlying mechanism of Rghl/Mla evolution might not be characterized by simple accumulation of nonsynonymous substitutions. Rather, this process probably also involved a recombination event that swapped individual domains or even gene transfer from closely related species. Consistent with this idea, in the topology of RGH1/MLA in the neighbor-net analysis, the separation of each subfamily is clearer compared with the corresponding NJ tree (Figs. 1 and 2 ). The lack of detectable $B$. graminis hordei resistance activity in subfamily 2 deployed in cultivated barley might indicate that the responsible signaling pathway is absent or not effective in these varieties. This implies that the two Mla subfamilies have functionally diverged from each other. It should be noted that overexpression of a barley MLA chimera containing CC and NB-ARC domains of MLA25-1, a subfamily 2 member of unknown function, fused to the LRR of barley MLA1, a subfamily 1 member, retains MLA1dependent resistance to $B$. graminis hordei (Jordan et al. 2011). This finding might suggest that subfamily 1 and 2 CC domains can be interchanged without affecting effector recognition. Unlike the chimera consisting of MLA25-1 CC-NBARC and MLA1 LRR, the chimera containing MLA18-1 CC-NB-ARC and MLA1 LRR does not exhibit MLA1-dependent resistance to $B$. graminis hordei even when overexpressed (Jordan et al. 2011). This discrepancy suggests diversity in the downstream signaling pathways engaged by these two $\mathrm{CC}$ domains in cultivated barley. Together, this demonstrates that subfamily $2 \mathrm{CC}$ and NB-ARC domains can be functional in $B$. graminis hordei resistance when overexpressed but does not exclude the possibility that, in wild barley populations with native Mla gene expression levels, immunity to $B$. graminis hordei mediated by the $\mathrm{CC}$ of subfamily 2 members is generally inefficient in limiting pathogen reproduction.

Consistent with extensive $\mathrm{CC}$ diversification in the phylogeny of MLA subfamilies 1 and 2, the corresponding sequence alignment reveals 22 residues that are differentially occupied by charged or noncharged amino acids in the two subfamilies. These charged residues are likely surface-localized (Casey et al. 2016; Maekawa, et al. 2011a), thereby altering the surface charge distribution of the signaling module. Interestingly, the CC domains of MLA10 (subfamily 1) but not MLA18-1 and MLA25-1 (both subfamily 2) interact with barley WRKY transcription factors that repress immune responses to powdery mildew fungi (Jordan et al. 2011; Shen et al. 2007). The differential capacity of CC domains of subfamily 1 and 2 to interact with WRKYs implies diversified molecular function of the CC domains of the two subfamilies. The N-terminal 46 amino acids of the barley MLA10 CC domain, including three of the aforementioned 13 charge alterations, are critical for this interaction (Shen et al. 2007). Therefore, while the CC of MLA subfamily 1 is predicted to de-repress immune responses via direct interaction with these WRKYs (Shen et al. 2007), the CC of MLA subfamily 2 members might interact with other host proteins that are not present in cultivated barley and interfere with the same transcriptional machinery or another cellular process conferring immunity and cell death. 


\section{The LRR as pathogen recognition determinant.}

The location and clustering of sites under positive selection for subfamily 1 is generally conserved between the MLA sequences from wild barley and functionally validated receptor variants conferring resistance to B. graminis hordei (Fig. 3). Notably, most positive selection sites are found on the surface of the predicted concave side of the LRR solenoid and these sites are mostly shared between the natural MLA variants. This implies that the previously reported sites under positive selection, which were detected among a set of MLA variants conferring B. graminis hordei resistance (Seeholzer et al. 2010), closely reflect the evolutionary pressure acting on this receptor region in wild barley populations. Our recent data suggest that MLA receptors directly recognize sequence-unrelated B. graminis hordei AVR effectors (I. M. L. Saur and S. Bauer unpublished data). Thus, the clustering of positively selected amino acid residues in the C-terminal LRRs of both MLA receptor subfamilies might define contact residues for direct effector interactions that do not compromise one or more conformational changes of the full-length receptor from the off state to the on state. The relevance of positive selection sites for direct interactions with AVR proteins has been shown for a subset of $\mathrm{L}$ resistance alleles in flax conferring immunity to the rust pathogen Melampsora linii (Dodds et al. 2006; Wang et al. 2007).

\section{Mining wild barley germplasm \\ for novel Mla resistance specificities.}

In wheat, the Pm3 locus is the main source of genetically encoded disease resistance against the wheat powdery mildew fungus B. graminis f. sp. tritici (Krattinger and Keller 2016). Similar to barley Mla, 17 allelic variants of the $P m 3$ gene have been identified in wheat populations that determine isolatespecific immunity to $B$. graminis $\mathrm{f}$. sp. tritici carrying matching AVR genes (Krattinger and Keller 2016). Although Pm3 is widely deployed in domesticated hexaploid wheat (Triticum aestivum), in T. dicoccoides, the progenitor of most cultivated wheat species, high presence-absence variation of polymorphism in the Pm3 gene was observed as well as a low sequence diversity (61\% of 208 accessions lacked the Pm3 gene) (Sela et al. 2014; Srichumpa et al. 2005). This has been explained by a potential maintenance cost of $P m 3$ in T. dicoccoides in the absence of pathogen selection pressure. Consequently, it is thought that the observed functional diversification in $\mathrm{Pm} 3$ has occurred primarily after wheat domestication approximately 10,000 years ago (Sela et al. 2014; Srichumpa et al. 2005). This contrasts with the detection of Mla transcripts encoding sequence-diversified full-length MLA immune receptors in at least 40 of 50 tested wild barley accessions. This and the fact that at least some characterized Mla recognition specificities to $B$. graminis hordei in cultivated barley are derived from wild barley or barley landraces might help to explain why 25 functionally validated Mla recognition specificities to B. graminis hordei exhibit, on average, $>91 \%$ sequence identity, whereas 17 deduced allelic wheat $\mathrm{Pm} 3$ receptors share $>97 \%$ sequence identity (Seeholzer et al. 2010). Accordingly, continuous B. graminis hordei selection pressure and a much longer evolutionary time span has been available for Mla functional diversification in wild barley and this could account for the greater sequence diversity of MLA compared with Pm3 NLRs. Several novel natural Mla sequences from wild barley are distributed across the Mla phylogenetic tree, with functionally validated Mla resistance specificities to B. graminis hordei confined to subfamily 1. A conspicuous clustering of Mla resistance specificities is seen in a sublineage of subfamily 1 containing 13 known resistance specificities, including but not limited to Mla6, Mla7, Mla9, and Mla10 (Fig.
1). The identification of four novel Mla sequence variants from wild barley belonging to this sublineage makes these receptor variants prime candidates for future targeted disease resistance assays. Such experiments could be used to examine whether the receptor variants exhibit overlapping recognition specificities with known MLA receptors or to detect novel $B$. graminis hordei $A V R_{A}$ genes.

\section{MATERIALS AND METHODS}

\section{RNA sequencing.}

Total mRNA from barley plants was obtained from the first or second leaves at 16 to $19 \mathrm{~h}$ after challenge with B. graminis $\mathrm{f}$. sp. hordei, using the RNeasy plant mini kit (Qiagen). RNA-Seq libraries were prepared by the Max Planck Genome Center Cologne (Germany), using the Illumina TruSeq stranded RNA sample preparation kit, and were subjected to 150-bp pairedend sequencing, using the Illumina HiSeq2500 sequencing system. The RNA-Seq data generated for this study have been deposited in the National Center for Biotechnology Information Sequence Read Archive (SRA) database (BioProject accession number PRJNA432492, SRA accession number SRP132475).

\section{De novo transcriptome assembly.}

For each barley accession, a de novo transcriptome assembly was performed using Trinity (version 2.2.0) (Grabherr et al. 2011) with default parameter settings for paired-end reads; transcript abundance was subsequently estimated (with options --est_method RSEM --algn_method bowtie), and peptide sequences of the best-scoring open reading frames (ORFs) were extracted using TransDecoder (Haas et al. 2013).

\section{Extraction of candidate MLA sequences.}

To identify candidate MLA sequences, BLAST searches were performed for the assembled transcripts (from Trinity) and predicted peptides (from TransDecoder) against the coding sequences (CDS) and protein sequences of the previously described Mla recognition specificities (Seeholzer et al. 2010) with $\mathrm{E}$ value cutoffs of $1 \mathrm{e}^{-6}$. For each sample, we identified the Trinity transcript group that contained the best BLAST hit (based on score, identity, and alignment length) against MLA. From this transcript group, a representative candidate MLA transcript was extracted manually, based on the BLAST statistics (score, identity, alignment length), transcript abundance (RSEM), inspection of transcript read coverage in the Integrative Genomics Viewer (IGV) (Robinson et al. 2011), and inspection of a Clustal Omega (Sievers et al. 2011) MSA of the identified and known MLA sequences. Based on this manual inspection, potential sequence errors were corrected, according to the RNA-Seq read alignment consensus (IGV visualization), and split sequences were merged as appropriate, based on the RNA-Seq read alignment and MSA. Subsequently, the corrected transcripts were used to extract corresponding bestscoring ORFs with TransDecoder, and RNA-Seq reads were mapped against the corrected sequences using bowtie2 (version 2.2.8) (Langmead and Salzberg 2012) with default parameters. The resulting alignments were again inspected in IGV and remaining sequence errors were corrected when required. This procedure of alternating read alignment and ORF prediction and manual screening and error correction was repeated until the RNA-Seq read consensus conformed to the predicted transcript sequence. In the end, the final candidate Mla/MLA CDS and peptide sequences were extracted from these corrected transcript sequences with TransDecoder. Accession numbers for the Mla/MLA sequences from wild barley are listed in Supplementary Table S4. 


\section{Phylogenetic analyses.}

The previously published sequences included in the phylogenetic analyses were retrieved from the National Center for Biotechnology Information (NCBI). As the NCBI accessions for MLA7 (AAQ55540) and MLA10 (AAQ55541) exhibit atypical amino acid variations compared to the previously obtained sequences (Maekawa et al. 2011; Seeholzer et al. 2010), for these two MLAs, we used the corresponding previously published sequences instead of the NCBI entries. The closest MLA homolog in Brachypodium distachion was identified by a BLAST search of the MLA consensus sequence against the Brachypodium sequences in the nonredundant NCBI protein database. The MLA homologs in barley 'Morex' were extracted by BLAST searches of the MLA CC domain against the updated 'Morex' genome annotations (Mascher et al. 2017) (IPK Barley BLAST server).

The MSA visualizations were generated using Unipro UGENE (Okonechnikov et al. 2012) with Clustal Omega as an alignment tool. The MLA consensus sequence for this visualization was generated from the 25 previously published fulllength MLA protein sequences (Seeholzer et al. 2010). NJ and ML phylogenetic trees were generated using MEGA5 (Tamura et al. 2011). Neighbor-net network analysis was performed using SplitsTree4 (applying "proteinMLdist" model: JTT) (Huson and Bryant 2006). CC domain haplotypes were extracted from an MSA with Clustal Omega. Tanglegrams were generated from the MEGA5 trees (after midpoint rooting), using the 'tanglegram' function of the R package 'dendextend' (Galili 2015) with previous stepwise greedy rotation to untangle the trees (using function 'untangle' with method "step2side").

For the phylogenetic analyses of individual MLA domains, we regarded the first $\mathrm{N}$-terminal 151 amino acids (1 to 151) as the CC domain, the sequence stretching from amino acid 180 to 481 as the NB-ARC domain, and the sequence from amino acid 490 to the end as the LRR.

The analyses of nucleotide diversity and positive selection were performed on codon alignments of the CDS sequences generated in MEGA5 (using ClustalW as an alignment tool). Nucleotide diversity was then calculated from these alignments, using the "nuc.div' function in the R package 'pegas' (Paradis 2010). Sites under episodic positive selection were identified using MEME (Murrell et al. 2012), with default parameters, and sites under pervasive positive selection were identified using FUBAR (Murrell et al. 2013), with default settings.

\section{Generation of expression constructs.}

All CC domains were synthesized by the GeneArt gene synthesis service (Thermo Scientific) as pDONR221 entry clones and were transferred into the pXCSG-GW-mYFP (monomeric yellow fluorescent protein) expression vector (Garcia et al. 2010) using LR Clonase II (Thermo Scientific). The resulting expression constructs were examined by Sanger sequencing (Eurofins).

\section{Agrobacterium-mediated transient transformation of Nicotiana benthamiana leaves.}

Agrobacterium tumefaciens GV3101 pMP90K were freshly transformed with the respective constructs of interest and were grown from single colonies in liquid Luria broth medium containing appropriate antibiotics for about $24 \mathrm{~h}$ at $28^{\circ} \mathrm{C}$. Bacterial cells were harvested by centrifugation at $2,500 \times g$ for $15 \mathrm{~min}$, followed by resuspension in infiltration medium $(10 \mathrm{mM}$ morpholineethanesulfonic acid, $\mathrm{pH} 5.6$, $10 \mathrm{mM} \mathrm{MgCl} 2$, and $200 \mu \mathrm{M}$ acetosyringone) to a final optical density at $600 \mathrm{~nm}=1.0$. Cultures were incubated for 2 to $4 \mathrm{~h}$ at $28^{\circ} \mathrm{C}$, with agitation at $180 \mathrm{rpm}$, before infiltration into leaves of three- to five-week-old $N$. benthamiana plants. Cell death was assessed 3 days postinfiltration. Experiments were repeated three times, independently, and a representative image is shown.

\section{Plant protein extraction and fusion protein detection by immunoblotting.}

Plant proteins were extracted as described previously (Saur et al. 2015) with the addition of IGEPAL (Sigma) at a final concentration of $0.25 \%$ to the protein extraction buffer. Extracts were diluted 4:1 with $4 \times$ Laemmli buffer (Bio-Rad) and were heated to $95^{\circ} \mathrm{C}$ for $5 \mathrm{~min}$. Samples were separated on $10 \%$ sodium dodecyl sulfate-polyacrylamide electrophoresis gels, were blotted onto polyvinylidene diflouride membranes, and were probed with anti-green fluorescent protein (abcam ab6556), followed by anti-rabbit IgG-HRP (horseradish peroxidase) (Santa Cruz Biotechnology) secondary antibodies. Proteins were detected by HRP activity on SuperSignal Femto chemiluminescent substrate (Thermo Fisher) using a Gel Doc $\mathrm{XR}+$ gel documentation system (Bio-Rad). Experiments were repeated three times, independently, and a representative image is shown.

\section{ACKNOWLEDGMENTS}

We thank the Max Planck Genome Center Cologne for RNA-Seq, P. Köchner and S. Haigis for technical assistance, and N. Donnelly for editing the manuscript. We thank M. J. Moscou for sharing unpublished data. We also thank J. Chai and R. Garrido-Oter for helpful suggestions.

\section{LITERATURE CITED}

Anderson, C., Khan, M. A., Catanzariti, A. M., Jack, C. A., Nemri, A., Lawrence, G. J., Upadhyaya, N. M., Hardham, A. R., Ellis, J. G., Dodds, P. N., and Jones, D. A. 2016. Genome analysis and avirulence gene cloning using a high-density RADseq linkage map of the flax rust fungus, Melampsora lini. BMC Genomics 17:667.

Axtell, M. J., and Staskawicz, B. J. 2003. Initiation of RPS2-specified disease resistance in Arabidopsis is coupled to the AvrRpt2-directed elimination of RIN4. Cell 112:369-377.

Bakker, E. G., Toomajian, C., Kreitman, M., and Bergelson, J. 2006. A genome-wide survey of $R$ gene polymorphisms in Arabidopsis. Plant Cell 18:1803-1818.

Caldo, R. A., Nettleton, D., and Wise, R. P. 2004. Interaction-dependent gene expression in Mla-specified response to barley powdery mildew. Plant Cell 16:2514-2528.

Casey, L. W., Lavrencic, P., Bentham, A. R., Cesari, S., Ericsson, D. J., Croll, T., Turk, D., Anderson, P. A., Mark, A. E., Dodds, P. N., Mobli, M., Kobe, B., and Williams, S. J. 2016. The CC domain structure from the wheat stem rust resistance protein $\mathrm{Sr} 33$ challenges paradigms for dimerization in plant NLR proteins. Proc. Natl. Acad. Sci. U.S.A. 113:12856-12861.

Cesari, S., Moore, J., Chen, C., Webb, D., Periyannan, S., Mago, R., Bernoux, M., Lagudah, E. S., and Dodds, P. N. 2016. Cytosolic activation of cell death and stem rust resistance by cereal MLA-family CC-NLR proteins. Proc. Natl. Acad. Sci. U.S.A. 113:10204-10209.

Cesari, S., Thilliez, G., Ribot, C., Chalvon, V., Michel, C., Jauneau, A., Rivas, S., Alaux, L., Kanzaki, H., Okuyama, Y., Morel, J. B., Fournier, E., Tharreau, D., Terauchi, R., and Kroj, T. 2013. The rice resistance protein pair RGA4/RGA5 recognizes the Magnaporthe oryzae effectors AVR-Pia and AVR1-CO39 by direct binding. Plant Cell 25:1463-1481.

Chen, J., Upadhyaya, N. M., Ortiz, D., Sperschneider, J., Li, F., Bouton, C., Breen, S., Dong, C., Xu, B., Zhang, X., Mago, R., Newell, K., Xia, X., Bernoux, M., Taylor, J. M., Steffenson, B., Jin, Y., Zhang, P., Kanyuka, K., Figueroa, M., Ellis, J. G., Park, R. F., and Dodds, P. N. 2017. Loss of $A v r S r 50$ by somatic exchange in stem rust leads to virulence for $\operatorname{Sr} 50$ resistance in wheat. Science 358:1607-1610.

Dodds, P. N., Lawrence, G. J., Catanzariti, A. M., Teh, T., Wang, C. I., Ayliffe, M. A., Kobe, B., and Ellis, J. G. 2006. Direct protein interaction underlies gene-for-gene specificity and coevolution of the flax resistance genes and flax rust avirulence genes. Proc. Natl. Acad. Sci. U.S.A. 103:8888-8893.

Ellis, J. G. 2016. Integrated decoys and effector traps: How to catch a plant pathogen. BMC Biol. 14:13. 
Flor, H. H. 1955. Host-parasite interaction in flax rusts-Its genetics and other implications. Phytopathology 45:680-685.

Galili, T. 2015. dendextend: An R package for visualizing, adjusting and comparing trees of hierarchical clustering. Bioinformatics 31 : 3718-3720.

García, A. V., Blanvillain-Baufumé, S., Huibers, R. P., Wiermer, M., Li, G., Gobbato, E., Rietz, S., and Parker, J. E. 2010. Balanced nuclear and cytoplasmic activities of EDS1 are required for a complete plant innate immune response. PLoS Pathog. 6:e1000970.

Glawe, D. A. 2008. The powdery mildews: A review of the world's most familiar (yet poorly known) plant pathogens. Annu. Rev. Phytopathol. 46:27-51.

Grabherr, M. G., Haas, B. J., Yassour, M., Levin, J. Z., Thompson, D. A., Amit, I., Adiconis, X., Fan, L., Raychowdhury, R., Zeng, Q., Chen, Z., Mauceli, E., Hacohen, N., Gnirke, A., Rhind, N., di Palma, F., Birren, B. W., Nusbaum, C., Lindblad-Toh, K., Friedman, N., and Regev, A. 2011. Full-length transcriptome assembly from RNA-Seq data without a reference genome. Nat. Biotechnol. 29:644-652.

Gutierrez, J. R., Balmuth, A. L., Ntoukakis, V., Mucyn, T. S., GimenezIbanez, S., Jones, A. M., and Rathjen, J. P. 2010. Prf immune complexes of tomato are oligomeric and contain multiple Pto-like kinases that diversify effector recognition. Plant J. 61:507-518.

Haas, B. J., Papanicolaou, A., Yassour, M., Grabherr, M., Blood, P. D., Bowden, J., Couger, M. B., Eccles, D., Li, B., Lieber, M., MacManes, M. D., Ott, M., Orvis, J., Pochet, N., Strozzi, F., Weeks, N., Westerman, R., William, T., Dewey, C. N., Henschel, R., LeDuc, R. D., Friedman, N., and Regev, A. 2013. De novo transcript sequence reconstruction from RNA-seq using the Trinity platform for reference generation and analysis. Nat. Protoc. 8:1494-1512.

Halterman, D. A., and Wise, R. P. 2004. A single-amino acid substitution in the sixth leucine-rich repeat of barley MLA6 and MLA13 alleviates dependence on RAR1 for disease resistance signaling. Plant J. 38:215-226.

Huson, D. H., and Bryant, D. 2006. Application of phylogenetic networks in evolutionary studies. Mol. Biol. Evol. 23:254-267.

Jacob, F., Kracher, B., Mine, A., Seyfferth, C., Blanvillain-Baufumé, S., Parker, J. E., Tsuda, K., Schulze-Lefert, P., and Maekawa, T. 2018. A dominant-interfering camta3 mutation compromises primary transcriptional outputs mediated by both cell surface and intracellular immune receptors in Arabidopsis thaliana. New Phytol. 217:1667-1680.

Jacob, F., Vernaldi, S., and Maekawa, T. 2013. Evolution and conservation of plant NLR functions. Front. Immunol. 4:297.

Jahoor, A., and Fischbeck, G. 1987. genetical studies of resistance of powdery mildew in barley lines derived from Hordeum spontaneum collected from Israel. Plant Breed. 99:265-273.

Jones, J. D., and Dangl, J. L. 2006. The plant immune system. Nature 444: 323-329.

Jordan, T., Seeholzer, S., Schwizer, S., Töller, A., Somssich, I. E., and Keller, B. 2011. The wheat Mla homologue TmMlal exhibits an evolutionarily conserved function against powdery mildew in both wheat and barley. Plant J. 65:610-621.

Jørgensen, J. H. 1994. Genetics of powdery mildew resistance in barley. Crit. Rev. Plant Sci. 13:97-119.

Karasov, T. L., Kniskern, J. M., Gao, L., DeYoung, B. J., Ding, J., Dubiella, U., Lastra, R. O., Nallu, S., Roux, F., Innes, R. W., Barrett, L. G., Hudson, R. R., and Bergelson, J. 2014. The long-term maintenance of a resistance polymorphism through diffuse interactions. Nature 512: 436-440.

Krattinger, S. G., and Keller, B. 2016. Molecular genetics and evolution of disease resistance in cereals. New Phytol. 212:320-332.

Langmead, B., and Salzberg, S. L. 2012. Fast gapped-read alignment with Bowtie 2. Nat. Methods 9:357-359.

Lu, X., Kracher, B., Saur, I. M., Bauer, S., Ellwood, S. R., Wise, R., Yaeno, T., Maekawa, T., and Schulze-Lefert, P. 2016. Allelic barley MLA immune receptors recognize sequence-unrelated avirulence effectors of the powdery mildew pathogen. Proc. Natl. Acad. Sci. U.S.A. 113: E6486-E6495.

Mackey, D., Holt, B. F., 3rd, Wiig, A., and Dangl, J. L. 2002. RIN4 interacts with Pseudomonas syringae type III effector molecules and is required for RPM1-mediated resistance in Arabidopsis. Cell 108:743-754.

Maekawa, T., Cheng, W., Spiridon, L. N., Töller, A., Lukasik, E., Saijo, Y., Liu, P., Shen, Q. H., Micluta, M. A., Somssich, I. E., Takken, F. L. W., Petrescu, A. J., Chai, J., and Schulze-Lefert, P. 2011a. Coiled-coil domain-dependent homodimerization of intracellular barley immune receptors defines a minimal functional module for triggering cell death. Cell Host Microbe 9:187-199.

Maekawa, T., Kufer, T. A., and Schulze-Lefert, P. 2011b. NLR functions in plant and animal immune systems: So far and yet so close. Nat. Immunol. 12:817-826.
Mago, R., Zhang, P., Vautrin, S., Šimková, H., Bansal, U., Luo, M. C., Rouse, M., Karaoglu, H., Periyannan, S., Kolmer, J., Jin, Y., Ayliffe, M. A., Bariana, H., Park, R. F., McIntosh, R., Doležel, J., Bergès, H., Spielmeyer, W., Lagudah, E. S., Ellis, J. G., and Dodds, P. N. 2015. The wheat $\mathrm{Sr} 50$ gene reveals rich diversity at a cereal disease resistance locus. Nat. Plants 1:15186.

Maqbool, A., Saitoh, H., Franceschetti, M., Stevenson, C. E., Uemura, A., Kanzaki, H., Kamoun, S., Terauchi, R., and Banfield, M. J. 2015. Structural basis of pathogen recognition by an integrated HMA domain in a plant NLR immune receptor. eLife 4:e08709.

Mascher, M., Gundlach, H., Himmelbach, A., Beier, S., Twardziok, S. O., Wicker, T., Radchuk, V., Dockter, C., Hedley, P. E., Russell, J., Bayer, M., Ramsay, L., Liu, H., Haberer, G., Zhang, X. Q., Zhang, Q., Barrero, R. A., Li, L., Taudien, S., Groth, M., Felder, M., Hastie A., Šimková, H., Staňková, H., Vrána, J., Chan, S., MuñozAmatriaín, M., Ounit, R., Wanamaker, S., Bolser, D., Colmsee, C., Schmutzer, T., Aliyeva-Schnorr, L., Grasso, S., Tanskanen, J., Chailyan, A., Sampath, D., Heavens, D., Clissold, L., Cao, S., Chapman, B., Dai, F., Han, Y., Li, H., Li, X., Lin, C., McCooke, J. K., Tan, C., Wang, P., Wang, S., Yin, S., Zhou, G., Poland, J. A., Bellgard, M. I., Borisjuk, L., Houben, A., Doležel, J., Ayling, S., Lonardi, S., Kersey, P., Langridge, P., Muehlbauer, G. J., Clark, M. D., Caccamo, M., Schulman, A. H., Mayer, K. F. X., Platzer, M., Close, T. J., Scholz, U., Hansson, M., Zhang, G., Braumann, I., Spannagl, M., Li, C., Waugh, R., and Stein, N. 2017. A chromosome conformation capture ordered sequence of the barley genome. Nature 544:427-433.

Murrell, B., Moola, S., Mabona, A., Weighill, T., Sheward, D., Kosakovsky Pond, S. L., and Scheffler, K. 2013. FUBAR: A fast, unconstrained Bayesian approximation for inferring selection. Mol. Biol. Evol. 30: 1196-1205.

Murrell, B., Wertheim, J. O., Moola, S., Weighill, T., Scheffler, K., and Kosakovsky Pond, S. L. 2012. Detecting individual sites subject to episodic diversifying selection. PLoS Genet. 8:e1002764.

Ntoukakis, V., Balmuth, A. L., Mucyn, T. S., Gutierrez, J. R., Jones, A. M., and Rathjen, J. P. 2013. The tomato Prf complex is a molecular trap for bacterial effectors based on Pto transphosphorylation. PLoS Pathog. 9: e1003123.

Okonechnikov, K., Golosova, O., and Fursov, M.; UGENE team. 2012. Unipro UGENE: A unified bioinformatics toolkit. Bioinformatics 28: 1166-1167.

Ortiz, D., de Guillen, K., Cesari, S., Chalvon, V., Gracy, J., Padilla, A., and Kroj, T. 2017. Recognition of the Magnaporthe oryzae effector AVR-Pia by the decoy domain of the rice NLR immune receptor RGA5. Plant Cell 29:156-168.

Pankin, A., Altmüller, J., Becker, C., and von Korff, M. 2018. Targeted resequencing reveals genomic signatures of barley domestication. New Phytol. 218:1247-1259.

Paradis, E. 2010. pegas: An R package for population genetics with an integrated-modular approach. Bioinformatics 26:419-420.

Periyannan, S., Moore, J., Ayliffe, M., Bansal, U., Wang, X., Huang, L., Deal, K., Luo, M., Kong, X., Bariana, H., Mago, R., McIntosh, R., Dodds, P., Dvorak, J., and Lagudah, E. 2013. The gene Sr33, an ortholog of barley Mla genes, encodes resistance to wheat stem rust race $\mathrm{Ug} 99$. Science 341:786-788.

Pillen, K., Zacharias, A., and Léon, J. 2003. Advanced backcross QTL analysis in barley (Hordeum vulgare L.). Theor. Appl. Genet. 107: 340-352.

Rairdan, G. J., Collier, S. M., Sacco, M. A., Baldwin, T. T., Boettrich, T. and Moffett, P. 2008. The coiled-coil and nucleotide binding domains of the potato Rx disease resistance protein function in pathogen recognition and signaling. Plant Cell 20:739-751.

Robinson, J. T., Thorvaldsdóttir, H., Winckler, W., Guttman, M., Lander, E. S., Getz, G., and Mesirov, J. P. 2011. Integrative Genomics Viewer. Nat. Biotechnol. 29:24-26.

Saur, I. M., Conlan, B. F., and Rathjen, J. P. 2015. The N-terminal domain of the tomato immune protein Prf contains multiple homotypic and Pto kinase interaction sites. J. Biol. Chem. 290:11258-11267.

Seeholzer, S., Tsuchimatsu, T., Jordan, T., Bieri, S., Pajonk, S., Yang, W., Jahoor, A., Shimizu, K. K., Keller, B., and Schulze-Lefert, P. 2010. Diversity at the Mla powdery mildew resistance locus from cultivated barley reveals sites of positive selection. Mol. Plant-Microbe Interact 23: 497-509.

Sela, H., Spiridon, L. N., Ashkenazi, H., Bhullar, N. K., Brunner, S., Petrescu, A. J., Fahima, T., Keller, B., and Jordan, T. 2014. Threedimensional modeling and diversity analysis reveals distinct AVR recognition sites and evolutionary pathways in wild and domesticated wheat Pm3 R genes. Mol. Plant-Microbe Interact 27:835-845. 
Shen, Q. H., Saijo, Y., Mauch, S., Biskup, C., Bieri, S., Keller, B., Seki, H., Ulker, B., Somssich, I. E., and Schulze-Lefert, P. 2007. Nuclear activity of MLA immune receptors links isolate-specific and basal diseaseresistance responses. Science 315:1098-1103.

Shen, Q. H., Zhou, F., Bieri, S., Haizel, T., Shirasu, K., and Schulze-Lefert, P. 2003. Recognition specificity and RAR1/SGT1 dependence in barley Mla disease resistance genes to the powdery mildew fungus. Plant Cell 15:732-744.

Sievers, F., Wilm, A., Dineen, D., Gibson, T. J., Karplus, K., Li, W., Lopez, R., McWilliam, H., Remmert, M., Söding, J., Thompson, J. D., and Higgins, D. G. 2011. Fast, scalable generation of high-quality protein multiple sequence alignments using Clustal Omega. Mol. Syst. Biol. 7:539.

Srichumpa, P., Brunner, S., Keller, B., and Yahiaoui, N. 2005. Allelic series of four powdery mildew resistance genes at the Pm3 locus in hexaploid bread wheat. Plant Physiol. 139:885-895.

Stahl, E. A., Dwyer, G., Mauricio, R., Kreitman, M., and Bergelson, J. 1999. Dynamics of disease resistance polymorphism at the Rpm1 locus of Arabidopsis. Nature 400:667-671.

Tameling, W. I., Vossen, J. H., Albrecht, M., Lengauer, T., Berden, J. A., Haring, M. A., Cornelissen, B. J., and Takken, F. L. 2006. Mutations in the NB-ARC domain of I-2 that impair ATP hydrolysis cause autoactivation. Plant Physiol. 140:1233-1245.

Tamura, K., Peterson, D., Peterson, N., Stecher, G., Nei, M., and Kumar, S. 2011. MEGA5: Molecular evolutionary genetics analysis using maximum likelihood, evolutionary distance, and maximum parsimony methods. Mol. Biol. Evol. 28:2731-2739.

Tian, D., Traw, M. B., Chen, J. Q., Kreitman, M., and Bergelson, J. 2003. Fitness costs of $R$-gene-mediated resistance in Arabidopsis thaliana. Nature 423:74-77.

Wang, C. I., Guncar, G., Forwood, J. K., Teh, T., Catanzariti, A. M., Lawrence, G. J., Loughlin, F. E., Mackay, J. P., Schirra, H. J., Anderson,
P. A., Ellis, J. G., Dodds, P. N., and Kobe, B. 2007. Crystal structures of flax rust avirulence proteins AvrL567-A and -D reveal details of the structural basis for flax disease resistance specificity. Plant Cell 19: 2898-2912.

Wang, H. 2005. AB-QTL analysis for two populations of winter barley sharing the donor of Hordeum vulgare ssp. spontaneum. Ph.D. dissertation, University of Bonn, Germany.

Wei, F., Gobelman-Werner, K., Morroll, S. M., Kurth, J., Mao, L., Wing, R., Leister, D., Schulze-Lefert, P., and Wise, R. P. 1999. The Mla (powdery mildew) resistance cluster is associated with three NBS-LRR gene families and suppressed recombination within a 240-kb DNA interval on chromosome 5S (1HS) of barley. Genetics 153:1929-1948.

Wei, F., Wing, R. A., and Wise, R. P. 2002. Genome dynamics and evolution of the Mla (powdery mildew) resistance locus in barley. Plant Cell 14: 1903-1917.

Zhou, F., Kurth, J., Wei, F., Elliott, C., Valè, G., Yahiaoui, N., Keller, B., Somerville, S., Wise, R., and Schulze-Lefert, P. 2001. Cell-autonomous expression of barley Mlal confers race-specific resistance to the powdery mildew fungus via a Rarl-independent signaling pathway. Plant Cell 13:337-350.

Zhu, M., Jiang, L., Bai, B., Zhao, W., Chen, X., Li, J., Liu, Y., Chen, Z., Wang, B., Wang, C., Wu, Q., Shen, Q., Dinesh-Kumar, S. P., and Tao, X. 2017. The intracellular immune receptor $\mathrm{Sw}-5 \mathrm{~b}$ confers broad-spectrum resistance to tospoviruses through recognition of a conserved 21-amino acid viral effector epitope. Plant Cell 29:2214-2232.

\section{AUTHOR-RECOMMENDED INTERNET RESOURCE}

The IPK Barley BLAST server: http://webblast.ipk-gatersleben.de/barley_ibsc 